\title{
Progression wird verlangsamt
}

_ Die Zahl der Patienten mit autosomaldominanter polyzystischer Nierenerkrankung (ADPKD) liegt in Deutschland bei etwa 60.000-80.000. Ursache für diese häufige Erbkrankheit ist, wie Prof. Nicholas Obermüller, Frankfurt/Main ausführte, eine Mutation in den Genen PKD1 oder PKD2: Sie führt zur Hochregulation von zyklischem Adenosinmonophosphat (cAMP), was durch Vasopressin noch weiter verstärkt wird. Die Folge ist die Bildung und ständige Vergrößerung von Nierenzysten bis hin zur terminalen Niereninsuffizienz. Diese tritt in der Regel mit 50-60 Jahren ein.

\section{Nierenfunktion länger erhalten}

Prof. Peter Gross, Dresden, präsentierte die TEMPO-3/4-Studie, in der 1.445 erwachsene ADPKD-Patienten entweder Tolvaptan oder Placebo erhielten. Tolvap$\tan \left(\operatorname{Jinarc}^{\circledR}\right)$ blockiert spezifisch die Bindung von Vasopressin an den V2-Rezeptor des distalen Nephrons und hemmt damit die fehlerhafte Signalkaskade sowie Zystenbildung und -wachstum in den Nieren. Nach drei Jahren hatte das Nierenvolumen - dies war der primäre Endpunkt - in der Verumgruppe um 2,8\% pro Jahr zugenommen, vs. 5,5\% pro Jahr $(\mathrm{p}<0,001)$ in der Placebogruppe.

Der wichtigste sekundäre Endpunkt umfasste eine mindestens 25\%ige Verschlechterung der Nierenfunktion sowie Neuauftreten oder Verschlechterung von Nierenschmerzen, Hypertonie und Albuminurie. Auch dieser Endpunkt wurde, was die Nierenfunktion un die Nierenschmerzen betraf, unter Tolvaptan signifikant seltener erreicht, vor allem, weil die Nierenfunktion der Patienten besser erhalten blieb.

Durst und Polyurie sowie Brust- und Kopfschmerzen, Hämaturie und (reversible) Leberenzymerhöhungen traten unter Tolvaptan häufiger auf. Unter Placebo wurden dagegen öfter renale Zysteninfektionen und Pyelonephritis beobachtet.

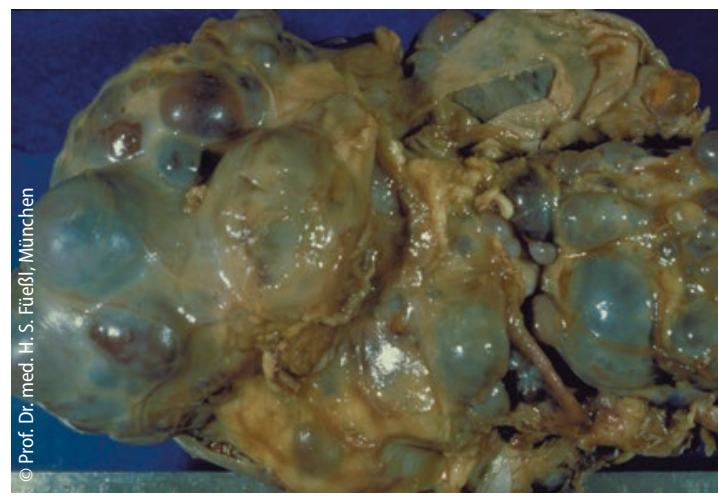

Dieses Endstadium sollte so lange wie möglich hinausgezögert werden.

Gross wies darauf hin, dass Tolvaptan wegen einer möglichen Lebertoxizität unter ärztlicher Aufsicht durchgeführt werden müsse. Er fasste zusammen: „Mit Jinarc ${ }^{\circledast}$ steht erstmals eine Therapie bei ADPKD zur Verfügung, mit der wir frühzeitig das Fortschreiten der Krankheit verlangsamen und eine terminale Niereninsuffizienz hinauszögern können.“

Simone Reisdorf

- Launch-Pressekonferenz „JINARC (Tolvaptan) - erste zielgerichtete Therapie der ADPKD“; Frankfurt/Main, Juni 2015 (Veranstalter:Otsuka)

\section{Diabetes mellitus}

\section{Erweiterte Zulassungen}

Der langjährig bewährte GLP-1-Rezeptor-Agonist Liraglutid kann nun auch bei moderater Niereninsuffizienz eingesetzt werden. Zu der Zulassungserweiterung wurden beim Diabeteskongress in Berlin Poster vorgestellt.

\section{Unerreichte $\mathrm{HbA}_{1 \mathrm{c}}$-Senkung}

„Kein anderes GLP-1-Analogon hat bisher eine stärkere $\mathrm{HbA}_{1 \mathrm{c}}$ - und Gewichtsreduktion erzeugt als Liraglutid“, berichtete Prof. Werner Kern, Ulm. Am meisten profitierten im klinischen Alltag laut Kern Patienten mit einer Diabetesdauer von bis zu fünf Jahren, die zuvor noch nicht viele andere Antidiabetika verwendet haben. Die positiven Ergebnisse bestätigten nun auch die 2-Jahres-Daten der Beobachtungsstudie Evidence mit über 3.000 Typ-2-Diabetikern [Gourdy P et al. $\mathrm{P} 239]$, und zwar mit einer $\mathrm{HbA}_{1 \mathrm{c}}$-Reduk- tion von bis zu 1,5\%-Punkten und einer Gewichtsabnahme von bis $\mathrm{zu} 3,2 \mathrm{~kg}$. Umso besser, so Kern, dass die Sicherheit und Wirksamkeit von Liraglutid (Victo$\mathrm{Za}^{\varpi}$ ) in der Standarddosis $1,8 \mathrm{mg} / \mathrm{d}$ nun auch bei Patienten mit moderater Niereninsuffizenz (GFR 30-59 ml/min/1,73 $\mathrm{m}^{2}$ ) belegt ist [Merker L et al. P35].

\section{Insulin detemir nun auch für Kinder ab dem Alter von einem Jahr}

Die Zulassungserweiterung basiert auf den Daten der Studie BEGIN ${ }^{\circledR}$ YOUNG 1 zur Wirksamkeit und Sicherheit von Insulin detemir (Levemir ${ }^{\circledR}$ ) [Thalange $\mathrm{N}$ et al. Pediatr Diabetes. 2015;16:164-76], in der die einmal oder zweimal tägliche Gabe von Insulin detemir in Kombination mit Insulin aspart auch für Kinder ab dem Alter von einem Jahr effektiv und gut verträglich war.

\section{Optimale Blutzuckereinstellung früh anstreben}

Das Erreichen einer guten Blutzuckereinstellung ist bei Kindern angesichts vielschichtiger Faktoren der Lebensweise und entwicklungsbedingter physiologischer Veränderungen auf dem Weg ins Erwachsenenalter eine Herausforderung. Ziele der Diabetestherapie bei Kindern sind die Vermeidung akuter Stoffwechselentgleisungen, die Prävention von Folgeerkrankungen sowie eine normale körperliche Entwicklung und altersentsprechende Leistungsfähigkeit. Hier könne, so Prof. Dr. Thomas Danne, Hannover, Insulin detemir einen wertvollen Beitrag leisten.

Sarah Louise Pampel

- Pressekonferenz ${ }_{\text {"Changing Diabetes }}{ }^{\oplus}$ - Herausforderungen einer Optimierung der Diabetesversorgung", bei der 50. Jahrestagung der Deutschen Diabetes-Gesellschaft; Berlin, Mai 2015 (Veranstalter: Novo Nordisk) 